\title{
MANIFESTATIONS OF A MASSIVE BLACK HOLE IN THE GALACTIC CENTER
}

\author{
E.S. PHINNEY \\ Theoretical Astrophysics \\ 130-33 Caltech \\ Pasadena, CA 91125 U.S.A.
}

\begin{abstract}
A young star cluster is a less contrived explanation than a massive black hole for many of the features seen in the Galactic center. However from a Copernican point of view, this explanation is less attractive than a black hole. The evidence for a $\sim 10^{6} \mathrm{M}_{\odot}$ black hole is becoming progressively less convincing, but the case against it is no stronger. We describe the development of a singular star cluster, as well as the processes of stellar disruption, merging, and gas accretion in such a cluster. Recently merged stars and tidally stripped giants may be detectable within an arcminute of the Galactic Center. We examine the physics of star formation in the inner parsecs of the galaxy, and the problem of maintaining the two parsec molecular torus.
\end{abstract}

\section{Introduction}

Those who argue that active galactic nuclei do not contain supermassive black holes are in the uncomfortable position of being apologists for models requiring extreme parameters or unconvincing physical assumptions, while the competition has the happier task of elaborating models already in qualitative agreement with the observed phenomena. The organizers have asked me to review the evidence for a massive $\left(\gtrsim 10^{4} \mathrm{M}_{\odot}\right)$ black hole in our own Galactic Nucleus. I must confess at the outset that the more I have thought about this topic, the more $I$ have become convinced that in the Galactic center, it is the big black holers who must be apologists, while those who believe in a "boring" star cluster have the easier task of explaining the observations. Within a Hubble time, however, such a star cluster would inexorably evolve through a collision-dominated phase, and it is hard to see how a massive black hole could avoid forming. The problem of quiet black holes is not unique to our Galaxy: the evidence for massive black holes in nearby inactive galaxies continues to mount. (Tonry, 1987, Kormendy, 1988a,b, Dressler \& Richstone, 1988). Reconciling lack of activity with an abundance of gas and a massive black hole is becoming less a branch of apologetics and more of a physical puzzle.

As in other galaxies exhibiting low levels of nuclear activity (Keel 1985) the central question is: Is the prime source of energy a cluster of massive stars forming at a rate much higher than the time-average, or is it accretion onto a massive black hole at a rate much lower than the time-average?

\section{The Coperuican Principle}

In neither time nor space should we be privileged observers of the universe. The predictions of this principle have been reliable guides for models of the solar system, the interstellar 


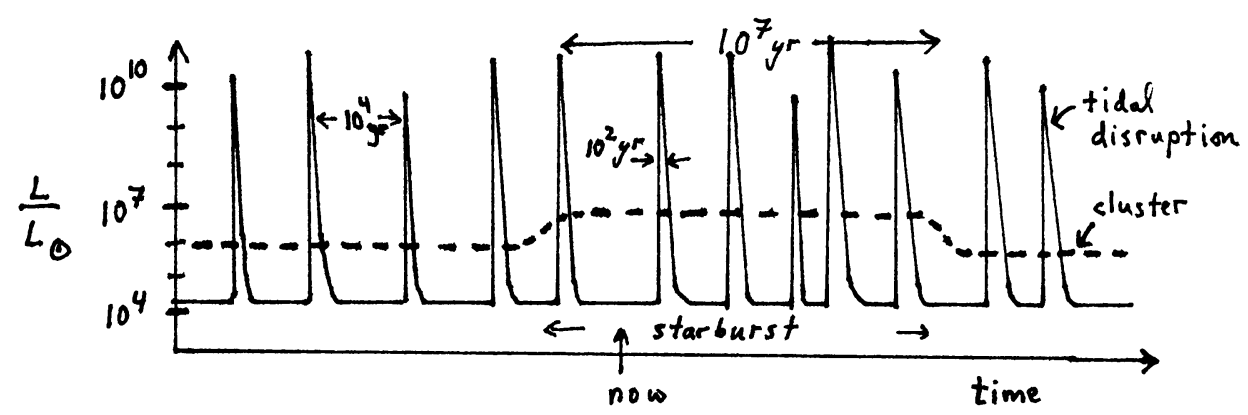

Figure 1 Luminosity $L$ of the Galactic Center, as a function of time. Both starburst and black hole models require similar $L(t)$, but in the former model the current state must be a peak, while in the latter a valley. Accretion onto a black hole is unlikely to produce the precently observed $10^{7} L_{\odot}$ in UV (sections 4.5 and 4.7). The unusual stars produced by runaway merging within $0.2 \mathrm{pc}$ (section 3.2) may allow the duty cycle of the starburst to be increased (section 2.1).

medium, and cosmology. We should therefore be suspicious of models of our Galactic center requiring an unusual or improbable state. The time dependence of models with a young star cluster and with a massive black hole are shown in figure 1.

A young cluster (Section 2.1) must now be seen at the (rare) peak of a burst. A black hole (Section 2.2) must now be in a (common) lull between bursts. As we shall see in sections 3-6, however, there are difficulties both for both models.

\subsection{STARBURST}

The mass within 2 parsecs (50") of the Galactic center is $M(<2 \mathrm{pc}) \sim 6 \times 10^{6} \mathrm{M}_{\odot}$ (Güsten, 1987, Rieke \& Rieke, 1988). The luminosity of late type stars in the inner 2 parsecs is $L_{\text {red }}(<$ $2 \mathrm{pc}) \sim 3 \times 10^{6} L_{\odot} \sim 2 \times 10^{-7} \mathrm{M}_{\odot} c^{2} \mathrm{yr}^{-1}$ (inferred from the observed $\left(\nu L_{\nu}\right)_{K}=3 \times 10^{4} L_{\odot}$ by assuming an extinction $A_{K} \sim 2.7$ (Lebofsky \& Rieke 1987) and applying a bolometric correction assuming the integrated spectrum is similar to that of other galactic spheroids, as first done by Becklin \& Neugebauer, 1968). A UV luminosity $L_{U V} \sim 10^{7} L_{\odot}$ is inferred from the radio continuum and recombination lines (Genzel \& Townes, 1987). The $\sim 250^{\circ} \mathrm{K}$ temperature of dust in the inner parsec (Gatley 1987) is consistent with $L_{U V} \sim 10^{7} L_{\odot}$, and in fact constrains the bolometric luminosity of the inner parsec to $\lesssim 4 \times 10^{7} L_{\odot}$. Stars liberate an energy of $\left(\epsilon_{H}=0.007\right) c^{2}$ per gram of hydrogen fused to helium (fusion of higher elements is energetically unimportant.) The mass of hydrogen burned in the lifetime of a star of mass $M$ solar masses is $M_{H b} \approx 0.13 M+[0.3+0.06 M]$, for $0.8<M<15$ (a crude fit; to the results of Renzini \& Buzzoni, 1986), where the first term is the mass burned on the "blue" main sequence, and the terms in braces give the mass burned in the "red" (beware Sk-69 202!) post-main sequence evolution.

Thus $f_{H}=M_{H b} / M$ varies from 0.5 for solar mass stars to 0.2 for $10 \mathrm{M}_{\odot} \mathrm{O} / \mathrm{B}$ stars, which live for $2 \times 10^{7}$ years. If stars produce all the luminosity $L$ from the inner 2 parsecs, the rate at which mass is being processed through stars is

$$
\dot{M}_{*}(<2 \mathrm{pc})=\frac{L(<2 \mathrm{pc})}{\epsilon_{H} f_{H} c^{2}} .
$$

If we include just $L_{\text {Red }}$, which could be due to an old population, we find $\dot{M}_{* \text {, Red }}(<2$ pc $)=$ $6 \times 10^{-5} \mathrm{M}_{\odot} \mathrm{yr}^{-1}$. In a Hubble time this corresponds to processing only $10 \%$ of the total 
mass-leaving a comfortable margin for additional mass in low-mass stars and the remnants of high-mass stars processed at earlier epochs. A more familiar way of saying the same thing is to note that with $A_{K} \sim 2.5$ the inner 2 parsecs of our Galaxy have $M / L_{K} \sim$ $2-3 \mathrm{M}_{\odot} / L_{K \odot}$, typical of the bulges of other spiral galaxies.

By contrast, $L_{U V} \sim 10^{7} L_{\odot}$ requires $\dot{M}_{*, U V}(<2 \mathrm{pc})=5 \times 10^{-4} \mathrm{M}_{\odot} \mathrm{yr}^{-1}$ and a supernova rate of $\sim 1 /\left(2 \times 10^{4} \mathrm{yr}\right)$ if the radiating stars end their lives as supernovae. If the IMF is like that in the solar neighborhood, that UV luminosity could not be sustained for more than $10 \%$ of the Hubble time without producing more than the observed mass. We would then be privileged observers. Trouble with the Copernican principle can be avoided only if the initial mass function is peaked at the mass of the $\sim 10 \mathrm{M}_{\odot}$ stars.

It should, however, be remembered that in the inner $0.5 \mathrm{pc}$ of the Galaxy, star-star collisions and tidal encounters are so frequent that stars of unusual states and masses can be produced (section 3.2). These might be a few times more efficient in turning their mass into UV radiation than $10 \mathrm{M}_{\odot}$ stars, lessening the discomfort of the Copernican principle.

\subsection{BLACK HOLE}

As discussed in section 5 (see also Rees 1988), a black hole of mass $10^{6} M_{6} M_{\odot}$ which tidally captures a star (of whose mass it retains a fraction $\eta$ ) will remain Eddington limited for $\sim 10 \epsilon^{3 / 5} M_{6}^{-2 / 5} \mathrm{yr}$ as it accretes the debris with radiative efficiency $\epsilon$. It will exceed the present, $\sim 10^{7} L_{\odot}$ for $\sim 10^{3} \epsilon^{3 / 5} M_{6}^{1 / 5} \mathrm{yr}$. The mean time between captures cannot be less than $10^{4} \eta \mathrm{yr}$, and is probably $10^{4}-10^{5} \mathrm{yr}$ (section 5 ). The duty cycle for stellar disruptions to produce the observed UV luminosity is thus $\lesssim 3 \%$. However steady accretion of gas liberated in star-star collisions (section 3.2) and the observed cooling gas at $\sim 0.1-2 \mathrm{pc}$ could easily maintain an $\dot{M} \sim 10^{-6} \epsilon^{-1} M_{\odot} \mathrm{yr}^{-1}$. This is adequate to produce the observed luminosity, so the Copernican principle would be satisfied (see figure 1). However, the spectrum of radiation produced by such accretion may be too hard to be consistent with observed line ratios (section 4.5) and the infrared flux too high (section 4.7), so a star cluster (and a steady accretion rate $<10^{-6} \epsilon^{-1} M_{\odot} \mathrm{yr}^{-1}$ ) is probably still required.

\section{Stars}

\section{1. (IN)SIGNIFICANCE OF STELLAR DENSITY PROFILES}

The $2.2 \mu$ luminosity density in the Galaxy scales as $r^{-1.8}$ (Allen, 1987) from radii $r$ of order $200 \mathrm{pc}$ to within a parsec or less of the Galactic center. This scaling is close to the $r^{-7 / 4}$ of a relaxed cluster bound to a black hole (Bahcall \& Wolf, 1976). Yet for $r>1 \mathrm{pc}$, the stars are clearly not bound to any central point mass. In M32, the optical luminosity density scales as $r^{-2.2}$ (Tonry, 1987) from $r \sim 100 \mathrm{pc}$ to $r<1 \mathrm{pc}$. This scaling is close to the $r^{-2.23}$ expected of a cluster after core-collapse (Inagaki \& Lynden-Bell 1983). Yet for $r>1 \mathrm{pc}$, the timescale for core collapse exceeds the Hubble time (by 4 orders of magnitude at $r \sim 100 \mathrm{pc}$ ). Within uncertainties of reddening corrections and population gradients, neither of these profiles can be distinguished from the $\rho_{*} \propto r^{-2}$ of a relaxed singular isothermal sphere. But for $r>10 \mathrm{pc}$, even the two-body relaxation time exceeds the Hubble time.

Conclusion: The murky methods of gas cooling and star formation that build spheroids must be able to forge the signatures of stellar-dynamical processes. When we see these signatures at radii where it is not crazy to invoke a black hole or core collapse, we should not be too hasty in accepting the profiles as evidence for either of these processes. 


\subsection{DEMOLITION DERBY}

Whether or not there is a black hole in the Galactic center, the region within $0.5 \mathrm{pc}$ $\left(12^{\prime \prime}\right)$ is a very hostile environment for stars. A majority of main sequence stars within this radius will have undergone collisional mergers. In a star cluster of one-dimensional velocity dispersion $\sigma$, the relative velocities $u$ of stars with a Maxwellian velocity distribution are also Maxwellian, with dispersion $\sqrt{2} \sigma$ (variances add!). Hence, accounting for gravitational focussing, the rate at which a given star, mass $M$, passes within a distance $d$ of stars, mass $m$, drawn from a population of density $n_{*}$ is

$$
\frac{1}{t(d)}=\frac{n_{*} \sqrt{\pi}}{2 \sigma^{3}} \int_{0}^{\infty} e^{-u^{2} / 4 \sigma^{2}}\left(u^{3} d^{2}+2 G(m+M) u d\right) d u
$$

For tidal captures between pairs of main sequence stars, $d \lesssim 2 R_{*}$, so most of the captures will result in mass loss and merging of the stars. Approximating $n_{*}$ by a singular isothermal sphere of dispersion $\sigma=80 \sigma_{80} \mathrm{~km} \mathrm{~s}^{-1}$, the stellar density at radius $r_{\mathrm{pc}} \mathrm{pc}$ is

$$
\rho_{*}=\frac{\sigma^{2}}{2 \pi G r^{2}}=2 \times 10^{5} M_{\odot} \mathrm{pc}^{-3} \sigma_{80}^{2} r_{\mathrm{pc}}^{-2}
$$

and the timescale $t_{m m}$ for merging of main sequence stars is given by

$$
\frac{1}{t_{m m}}=\frac{1}{2.5 \times 10^{11} \mathrm{yr}} \frac{\sigma_{80}}{r_{\mathrm{pc}}^{2}}\left(1+0.07 \sigma_{80}^{2}\right)
$$

Within a radius $\lesssim 0.2 \mathrm{pc}\left(5^{\prime \prime}\right.$ on the sky), a majority of turnoff-mass main sequence stars will have undergone direct collisions, probably resulting in mergers (Colgate 1967, Sanders 1970, Begelman \& Rees 1978, Lee \& Nelson 1988). The unusual blue stars making up IRS 16 may be such objects. Even at radii $\sim 5$ pc $\left(2^{\prime}\right.$ on the sky), there occurs roughly one merger every $10^{6}$ years, so several thousand such merged stars may exist there, two or three magnitudes brighter than the old turnoff stars.

Stars which survive the main sequence are likely to have their envelopes tidally stripped during the red giant phase. Fitting models of VandenBerg \& Laskarides (1987), we find that the time spent with radii larger than $R$ scales as $t(>R) \propto R^{-1.5}$ (time spent at luminosities $>L, t(>L) \propto L^{-0.85}$, and the temperature decreases slightly through the red giant phase). The rate of tidal stripping scales as $R^{2}$, so it is most likely to occur during the few million years at the tip of the giant branch, where $R \simeq 100 R_{\odot}$. Thus the probability of stripping during the giant phase is $\sim 0.02 \sigma_{80}^{3} r_{\mathrm{pc}}^{-2}$. Most giants within $\sim 0.2 \mathrm{pc}$ will be stripped.

Whether or not a massive black hole is present, the stellar population within $\sim 0.5 \mathrm{pc}$ of the Galactic Center (12" on the sky) is likely to be unusual. Any neutron stars or white dwarfs present have a high probability of capturing close binary companions and forming X-ray binaries and rapid pulsars. Large numbers of stars more massive than the turnoff mass will have been created by mergers, and red giant envelopes stripped. At larger radii, there should exist several hundred recent mergers still cooling onto their new main sequence. Furthermore, these tidal encounters between stars will result in the ejection of some fraction of the mass at speeds of up to a few hundred $\mathrm{km} \mathrm{s}^{-1}$. 


\subsection{INEXORABLE RELAXATION}

Could the Galactic Center have avoided this demolition derby? The core radius of the distribution of population II stars in the Galactic center has been variously estimated as $<0.1 \mathrm{pc}$ or $\sim 0.6 \mathrm{pc}$ (Allen 1987, Rieke \& Rieke 1988). If the former is correct, collisions are occurring even now. If the latter we are seeing the Galactic Center at a special time: the two-body relaxation timescale at radius $r_{\mathrm{pc}}$ of a singular isothermal sphere is

$$
t_{\text {relax }}=3 \times 10^{9} \mathrm{yr} \sigma_{80} r_{p c}^{2} \frac{1 M_{\odot}}{\langle m\rangle}
$$

and the central relaxation time $t_{r c}$ of an isothermal sphere of core radius $r_{p c} \mathrm{pc}$ is about 6 times shorter. If all stars in the Galactic Center had masses of $1 M_{\odot}$, the timescale for relaxation to cause collapse to zero core radius would be $330 t_{r c}$ (Cohn 1980), or $6 \times 10^{10} \mathrm{yr}$ for a core radius of $0.6 \mathrm{pc}$. However, with realistic initial mass functions, the collapse time is more typically $\lesssim 60 t_{r c}$ (Inagaki \& Saslaw 1985, Murphy \& Cohn 1988) since the heavier stars sink to the center and collapse first. A core radius of $0.6 \mathrm{pc}$ would thus last for less than a Hubble time. If our Galactic Center has not yet reached central densities high enough to be dominated by collisions, it soon will!

\section{Unconvincing Evidence for a $\sim 10^{6} M_{\odot}$ Black Hole}

The evidence for a massive black hole at the Galactic Center is underwhelming and largely circumstantial. The scraps of evidence are presented below. We also give alternative explanations for these scraps, and indicate what would have to be observed to convince a skeptic that these were not correct.

\subsection{STELLAR KINEMATICS}

At radii $>1 \mathrm{pc}$, the velocity dispersion is roughly constant at $\sim 80 \mathrm{~km} \mathrm{~s}^{-1}$, consistent with the light profile and a normal M/L $\left(\rho_{*} \propto r^{-1.8}\right.$; Becklin \& Neugebauer (1968)). At radii $<1 \mathrm{pc}\left(24^{\prime \prime}\right)$, the most recent careful measurements show more of the same (Rieke \& Rieke 1988, McGinn et al. 1988, Allen 1987), with perhaps a slight rise in the velocity dispersion and a slight flattening of the density profile (controversial because of the difficulty of subtracting the few bright stars which dominate the light). The measurements peter out at $\sim 0.3 \mathrm{pc}$, and thus rule out black holes more massive than $\sim 10^{6} M_{\odot}$, but say nothing about a black hole of mass $\leq 10^{6} M_{\odot}$.

A skeptic would be convinced by a velocity dispersion of $\gtrsim 10^{3} \mathrm{~km} \mathrm{~s}^{-1}$, or even by a single star with such a velocity (too high to be produced by 3-body encounters in a cluster since it exceeds the escape speed from a main sequence or giant star).

\subsection{GAS KINEMATICS}

At radii of $1-3 \mathrm{pc}$, the gas velocities are $\sim 100-200 \mathrm{~km} \mathrm{~s}^{-1}$ (Genzel \& Townes 1987), consistent with motions in the cluster defined by the stellar velocity dispersion. Within $0.1 \mathrm{pc}$ of IRS 16C, gas velocities extend up to $\sim 700 \mathrm{~km} \mathrm{~s}^{-1}$ (Geballe et al. 1987). This is, of course, consistent with virial motions about a $\sim 10^{6} M_{\odot}$ black hole, but would equally well be explained by winds from the hot stars of IRS 16 (Allen presents evidence in these proceedings that these are Wolf-Rayet stars, known to have strong high-velocity winds), or the remains of a star tidally disrupted by an encounter with another star or with a black hole of mass much lower than $10^{6} M_{\odot}$ (secton 5.1). 
A skeptic of black holes would probably be convinced of the need for tidal disruption by a massive black hole or an accretion disk wind if gas with velocity $>10^{4} \mathrm{~km} \mathrm{~s}^{-1}$ were seen. Such speeds are not seen in stellar winds or supernovae (though they are seen in SS 433, and could be produced in the tidal disruption by a neutron star of a red giant's degenerate core).

\subsection{UNIQUE IIARD $\gamma$-RAY SOURCE}

The unusual hard $\gamma$-ray source near the Galactic Center during 1985-1987 has been convincingly resolved as 1740.7-2942 (Skinner et al., Kawai et al. 1988 and Cook, Prince, Skinner, these proceedings), at a projected distance of $150 \mathrm{pc}$ from the Galactic Center! Only a very weak source $\left(\sim 4 \times 10^{35} \mathrm{erg} \mathrm{s}^{-1}, 3-30 \mathrm{keV}\right.$, Skinner et al. 1987) remains possibly coincident $\left(<1^{\prime}\right)$ with the Galactic Center. Such a weak source could be produced by the corona of an accretion disk around a supermassive black hole, but could equally well be a pulsar or a feeble X-ray binary.

Fans of the latter would give up if the X-ray source flared to a luminosity $>10^{40} \mathrm{erg} \mathrm{s}^{-1}$, brighter than the Crab pulsar and the Eddington limit for stellar mass objects.

\subsection{UNIQUE $511 \mathrm{KEV} \mathrm{SOURCE}$}

Detections of positron annihilation photons from the direction of the Galactic Center have so far been made only with detectors of resolution of many degrees or more. Much of the flux is probably extended on scales of $10^{\circ}$, and some of the apparent variability due to differences in the angular resolution of detectors (Share et al. 1988). This extended emission might come from positrons released in radioactive decay of supernova ejecta, or from many unresolved low mass X-ray binaries (Kluźniak et al. 1988). The remaining variable component could be produced near a massive black hole, but could more naturally be produced by a stellar mass object, more compact and thus with higher optical depth for $\gamma \gamma \rightarrow e^{+} e^{-}$(see Phinney 1983, Ramaty \& Lingenfelter 1987, Lightman et al. 1987, Begelman et al. 1987 for some of the difficulties of simple massive black hole models). Whatever the source of positrons, the narrow line width and 6-month variability time indicates that they must annihilate in one of the cool $\left(<10^{5}{ }^{\circ} \mathrm{K}\right)$ dense $\left(>10^{5} \mathrm{~cm}^{-3}\right)$ clouds (Lingenfelter \& Ramaty 1983) found in abundance near the Galactic Center. As the positron source turns 'on' again, it will be exciting to learn from imaging detectors whether it is coincident with 1740.7-2942, the Galactic Center, or some other source.

Skeptics of massive black hole models for the variable positron flux would be convinced if both a) it were shown to arise in a cloud within $1 \mathrm{pc}$ of the Galactic Center and b) its flux correlated (with a lag and probably a sign reversal) with convincingly non-stellar (section 4.3) flares of the central hard $\gamma$-ray source.

\subsection{IONIZING UV SOURCE}

The ionization of gas in the central parsec of the Galaxy requires a source of $\sim 10^{7} L_{\odot}$ in ionizing UV radiation (Genzel \& Townes 1987), probably localized within a fraction of a parsec of IRS 16 (Wade et al. 1987). The total far infrared luminosity reradiated by dust is $\lesssim 10^{7} L_{\odot}$, and the dust seems to have covering factor $\gtrsim 0.5$, so there cannot be substantial luminosity in other spectral bands. The lore from the line ratios of the photoionized gas is that the color temperature of this ionizing radiation, $T_{c} \sim 35,000^{\circ} \mathrm{K}$ (Serabyn \& Lacy 1985). If the effective temperature $T_{\text {eff }} \sim T_{c}$ then setting $\sigma T^{4} 2 \pi R^{2}=L_{\mathrm{UV}}$, the radius of the radiating surface, $R \gtrsim 10^{13} \mathrm{~cm} \simeq 60 M_{6}^{-1} r_{g}$, where $r_{g}=G M c^{-2}$ is the gravitational radius of a black hole of mass $M=10^{6} M_{\odot}$. Since most of the energy in an accretion disk 
is liberated at $\lesssim 10 r_{g}$, we would expect either much higher energy photons (if $T_{c}>T_{\text {eff }}$, as required by detailed balance for thermal radiation processes) or a much lower luminosity. Only if $M \gtrsim 4 \times 10^{6} M_{\odot}$ would a thermal accretion disk produce $10^{7} L_{\odot}$ at the required $T_{c}$; such high masses appear to be ruled out by the stellar kinematics. The required accretion rates are so low that it is not clear that a geometrically thin, optically thick accretion disk would form (Rees et al. 1982). Nonthermal UV emission (which can have $T_{\text {eff }}>T_{c}$ and would thus be consistent with the expected smaller radii) is therefore a possibility, but only in contrived situations would this have the very steep UV spectrum which seems to be required by the diagnostic line ratios. Closer investigation of the robustness of those diagnostic ratios (generally more sensitive to ionization parameter than the shape of the input spectrum) would be valuable. Constraints on the infrared flux from the position of Sgr $A^{*}$ make it very unlikely that an accretion flux there could produce even a tiny fraction of the required UV unless our line of sight to the black hole is much more heavily obscured than the typical $A_{K} \simeq 2.7 \mathrm{mag}$ (section 4.7).

If the estimate of $T_{c}$ and/or the IR constraint is correct, a more natural explanation of the large radiating surface area is that the UV comes from a group of hot stars, perhaps IRS 16 (Allen 1987, and these proceedings).

Skeptics of a massive black hole would be convinced if the UV luminosity rose to $\gtrsim$ $10^{42} \mathrm{erg} \mathrm{s}^{-1}$ for more than a month (ruling out a supernova) - as it might well do sometime during the next ice age if the black hole is present and tidally disrupts a star.

\subsection{UNIQUE RADIO SOURCE-SGR A*}

The original and still most convincing identification for a massive black hole at the Galactic Center is the unique radio source $\mathrm{Sgr} \mathrm{A}^{*}$, whose spectrum rises as $\nu^{1 / 3}$ to at least $300 \mathrm{GHz}$ (Mezger, these proceedings), and whose brightness temperature $T_{B}>10^{9} \mathrm{~K}$ at GHz frequencies (Jauncey et al. 1988). The spectrum and brightness temperatures are typical of those seen in the cores of extragalactic radio sources. As many people have realized (Reynolds \& McKee 1980, Engelke 1988), the spectrum can be produced by an inhomogeneous synchrotron source such as a wind, jet, or extended corona, in which the energy densities in magnetic field and radiating particles scale approximately as $r^{-2}$. Then the radius $r$ at which synchrotron self-absorption occurs at frequency $\nu$ scales roughly as $r \propto \nu^{-1}$. Superposition of the self-absorbed spectra from each radius then produce the observed flat or rising spectrum. In this picture, the inferred photospheric size at $10 \mathrm{GHz}$ is $r(10 \mathrm{GHz}) \sim 10^{14} \mathrm{~cm}$ (a few times smaller than the observed scatter-broadened size at $8.4 \mathrm{GHz}$ - Jauncey et al 1988), and the minimum total pressure $p_{\min }\left(10^{14} \mathrm{~cm}\right) \sim 1 \mathrm{dyn} \mathrm{cm}^{-2}$. It is attractive to identify this source with the corona of an accretion flow (Rees 1987) since the characteristic pressure at radius $10^{14} r_{14} \mathrm{~cm}$ in an accretion flow with accretion rate $10^{-6} \dot{M}_{-6} M_{\odot} \mathrm{yr}^{-1}$, viscosity parameter $\alpha(<1)$, and thickness $h$ is

$$
p_{i} \sim 1 \dot{M}_{-6} \alpha^{-1} M_{6}^{1 / 2} r_{14}^{-5 / 2}(r / h) \text { dyn } \mathrm{cm}^{-2} .
$$

If this identification is correct, the corona could continue in to $\sim 3 r_{g}=5 \times 10^{11} M_{6} \mathrm{~cm}$, and the spectrum could continue to rise to $\sim 10^{14} \mathrm{M}_{6}^{-1} \mathrm{~Hz}$ (at higher frequencies the source would be optically thin at all radii), though the $K$-band luminosity, $\sim 10^{37} \mathrm{erg} \mathrm{s}^{-1}$, is possibly in conflict with the observed upper limits (section 4.7).

The luminosity $\left(\sim 10^{35} \mathrm{erg} \mathrm{s}^{-1}\right.$ at frequencies below $\left.300 \mathrm{GHz}\right)$ and minimum total energy flux (for a relativistically expanding source $4 \pi r^{2} c p_{\min } \sim 2 \times 10^{38} \mathrm{erg} \mathrm{s}^{-1}$ ) are, however, not so high that a stellar origin can be ruled out. 
Weak evidence supporting the interpretation of $\mathrm{Sgr} \mathrm{A}^{*}$ as a massive black hole is its low proper motion $v_{t}<40 \mathrm{~km} \mathrm{~s}^{-1}$ (Backer \& Sramek 1987), a factor of two less than the velocity dispersion of nearby stars. If limits on the proper motion could be pushed to $<1 \mathrm{~km} \mathrm{~s}^{-1}$, the interpretation as a massive black hole would be compelling. Until then, interpretation of the radio source as a pulsar moving through a dense $\left(\gtrsim 10^{6} \mathrm{~cm}^{-3}\right)$ cloud remains an attractive possibility. The star formation rates inferred from the UV luminosity (section 2.1) are high enough that it is not unlikely that a young ( $\lesssim 10^{4} \mathrm{yr}$ ) pulsar with the required $I \Omega \dot{\Omega}>10^{38} \mathrm{erg} \mathrm{s}^{-1}$ should be found near the Galactic Center. The uniqueness of the spectrum is not a strong objection - nowhere else in the Galaxy is it probable to find a rapidly spinning pulsar in a gas cloud with density $\gtrsim 10^{6} \mathrm{~cm}^{-3}$.

\subsection{DIFFICULTIES}

The most compelling of the arguments against a massive black hole (Ozernoy, these proceedings) is the presence of the clump of bright stars IRS $16 \mathrm{C}$ projected $1.5^{\prime \prime}(0.06 \mathrm{pc})$ from Sgr A* (Forrest et al. 1987, Allen, these proceedings). If these represent a physical clump of stars, this will be tidally disrupted by a black hole of mass $10^{6} M_{6} M_{\odot}$ at the position of Sgr $A^{*}$, unless the clump lies more than $1 M_{6}^{1 / 3}$ pc from the hole and is only accidentally projected close to it (probability $<1 \%$ ). Alternatively the stars could lie close to their projected distance, but the grouping of bright stars could be accidental (probability $\sim 10 \%$ for all of 3 or 4 to be on the same side of the black hole). It seems unlikely $(\sim 3 \%)$ that the clump could be a physical group near the projected distance, since the tidal disruption timescale is then only $\sim 10^{3.5} M_{6}^{-1 / 2} \mathrm{yr}$, and the Copernican principle suggests that such $\sim 10 M_{\odot}$ clumps cannot form more often than once every $10^{5} \mathrm{yr}$.

The lower limit on the $K$ magnitude of Sgr $A^{*}\left(m_{K}>11.4\right.$, Allen, 1987) gives (assuming $A_{K}=2.7 \mathrm{mag}$ ) an upper limit to the $K$-band flux of $\left(\nu L_{\nu}\right)_{K}=2.5 \times 10^{36} \mathrm{erg} \mathrm{s}^{-1}$, painfully low for any thermal (Lacy et al. 1982) or nonthermal (Rees et al. 1982) model of an accretion flow with bolometric luminosity $\gtrsim 10^{38} \mathrm{erg} \mathrm{s}^{-1}$, corresponding to a steady accretion rate $\lesssim 3 \times 10^{-9} \epsilon^{-1} M_{\odot} \mathrm{yr}^{-1}$. Given the high $\left(\gtrsim 10^{-6} M_{\odot} \mathrm{yr}^{-1}\right)$ probable rate of gas supply (section 5), very low radiative efficiencies are indicated, though not unexpected at these low accretion rates (Rees et al. 1982).

Both objections vanish if the black hole lies within IRC 16C, but the low proper motion of $\mathrm{Sgr} \mathrm{A}^{*}$ is then improbable $(\sim 1 \%)$, requiring lucky projection or a hole mass $<10^{4} M_{\odot}$.

\section{Black Hole Growth and Accretion}

\subsection{BULIMIA vs. ANOREXIA: TIDAL DISRUPTION AND CAPTURE}

A star, mass $M_{*}$, passing a black hole, mass $M_{p}$, will not be largely disrupted until the energy in the raised tide exceeds the binding energy of the star $=f G M_{*}^{2} / R_{*}$, i.e., until

$$
d<d_{\text {dis }}=R_{*}\left(\frac{k}{f}\right)^{1 / 6}\left(\frac{M_{p}}{M_{*}}\right)^{1 / 3},
$$

corresponding to $\delta r / R_{*} \sim(f / k)^{1 / 2} \gg 1$. Here $k$ is the constant of apsidal motion, and $l_{1} / f=0.3$ for convective stars, 0.02 for radiative stars. The dynamical time for the disrupted star is thus somewhat longer than the timescale on which the tidal force changes through the orbit, so tides will be dynamical, not static. The envelope of the star will begin to be overwhelmed by the hole already when $\delta r / R_{*} \gtrsim 1 / 4$, i.e., when $d \sim 2\left(M_{p} / M_{*}\right)^{1 / 3} R_{*}$. 


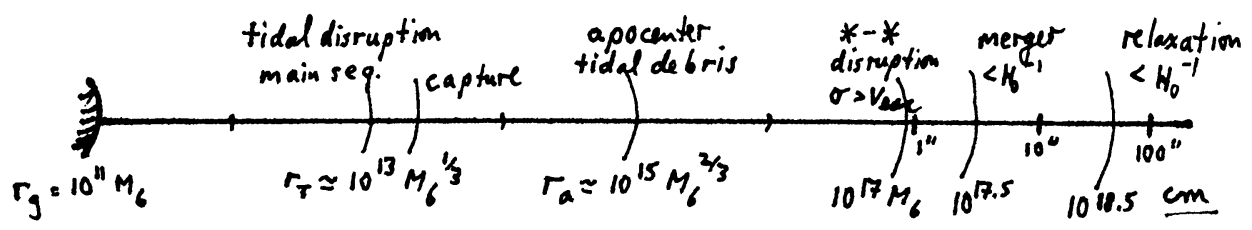

Figure 2 Significant radii for a stellar cluster, black hole, and tidal disruption events.

When the star is disrupted, the mean specific energy $e$ of the debris is reduced by $\sim G M_{*} / R_{*} \equiv v_{\text {esc }}^{2} / 2$, but the dispersion $\Delta e$ is much larger than the mean (Lacy et al. 1982), of order $\left(G M_{p} / d\right)\left(R_{*} / d\right) \sim\left(G M_{*} / R_{*}\right)\left(M_{p} / M_{*}\right)^{1 / 3}$. Thus roughly half of the star's mass is unbound, ejected with speed $\sim\left(M_{p} / M_{*}\right)^{1 / 6} v_{\text {esc }}$, while the other hall is bound, in orbits with apocentric radii $r_{a}$ as small as $R_{*}\left(M_{p} / M_{*}\right)^{2 / 3}$ (this effect is related to the 'gravitational slingshot' used by space probes, and to binary disruption, studied by Hills, 1988). Since $e=0$ is not special, $d M / d e$ should be roughly constant $\left(\sim M_{*} / \Delta e\right)$ near $-e=G M_{p} / r_{a} \simeq 0$, and the rate of fall-back accretion at time $T=\pi\left(r_{a}^{3} / 2 G M_{p}\right)^{1 / 2}$ after disruption

$$
\frac{d M}{d T}=\frac{d M}{d e} \frac{d e}{d r_{a}} \frac{d r_{a}}{d T} \simeq \frac{d M}{d e}\left(G M_{p}\right)^{2 / 3} T^{-5 / 3} .
$$

(This differs from Rees 1988, who gave $\dot{M} \propto T^{-5 / 2}$ ). Our understanding of accretion is inadequate to tell whether the black hole captures most of this gas, or ejects it in a wind (Rees, 1988, reviews the possibilities). The latter is certainly energetically possible: accretion of only $10^{-3} M_{6}^{1 / 3}$ of the disrupted material would suffice to unbind the rest.

The rate of tidal disruption is determined by the rate at which stars are scattered into the 'loss-cone' of orbits with pericentric distances less than $d_{\text {dis }}$. If we define the radius $r_{h}$ where the black hole begins to dominate the central cluster $r_{h}=G M_{p} / \sigma^{2}=0.7 M_{6} \sigma_{80}^{-2} \mathrm{pc}$, then we find that the loss cone can be filled outside radius $r_{f} \simeq 0.3 r_{h}\left(\sigma / v_{\text {esc }}\right)^{8 / 9} M_{6}^{4 / 27}$. Provided the stellar density at $r \gtrsim r_{f}$ scales roughly as $r^{-2}$, it is a good approximation to write the disruption rate as

$$
\dot{M}_{\mathrm{dis}} \sim 0.2 \frac{\sigma^{3}}{G} \frac{d_{\mathrm{dis}}}{r_{f}} \sim 10^{-3} \sigma_{80}^{4} M_{6}^{-0.8} M_{\odot} \mathrm{yr}^{-1}
$$

The rate could be $\sim 10 M_{6}^{-1.1}$ times lower if the star-star collisions discussed in section 3.2 flatten the density profile inside $\sim 0.3 \mathrm{pc}$. The rate of disruption of giant stars is lower than that of main sequence stars not only by the ratio of number densities, but also by a factor $\left(R_{\text {giant }} / R_{\odot}\right)^{5 / 9}$. Relevant radii are summarized in figure 2 .

\subsection{GAS SUPPLY}

Although a stellar-mass black hole in a collision-dominated cluster (or one with core-radius $>0.6 \mathrm{pc}$, section 3.3) with the minimal accretion fraction could avoid growth by stellar capture, one must still deal with accretion of gas. Bondi accretion of a hot intercloud phase $\left(n=10^{2} n_{2} \mathrm{~cm}^{-3}, T \sim 10^{6.3} \mathrm{~K}\right)$ confining the molecular tori at $\sim 1 \mathrm{pc}$ would give $\dot{M}_{B} \simeq 10^{-5} M_{6}^{q} M_{\odot} \mathrm{yr}^{-1}$ if the gas density scaled as $r^{2-q}$ at smaller radii. The collisional dissipation of the orbital energy of the molecular ring and ionized streamers gives $\dot{M}_{\text {edd }} \sim$ $10^{-3} M_{\odot} \mathrm{yr}^{-1}$. A cooling flow in the intercloud medium would give $\dot{M}_{c f} \simeq 10^{-4} n_{2}^{2} M_{\odot} \mathrm{yr}^{-1}$. 
As argued in section 3 , star-star collisions within $0.2 \mathrm{pc}$ are probably a significant further source of gas. The fate of all this gas may be (1) accretion onto a black hole - giving $\sim 10^{6} M_{\odot}$ in a Hubble time, (2) ejection, powered either by stellar processes or a black hole, or (3) star formation.

\section{The Fate of Gas}

\subsection{PROBLEMS OF STAR FORMATION}

The unusual luminous stars in and near the Galactic Center have been construed as evidence for recent star formation in the inner parsecs. There is also a substantial $\left(\sim 10^{-4} M_{\odot} \mathrm{yr}^{-1}\right)$ flux of gas (section 5) which must be disposed of. None of the directly observed gas can condense into stars. It could do so only if its number density $n$ exceeded the Roche density

$$
n_{\text {Roche }}=3 \times 10^{7} r_{p c}^{-2} \sigma_{80}^{2} \mathrm{~cm}^{-3} \text {. }
$$

Molecular clouds like those seen locally would be disrupted by the Galaxy's tidal field at $r \sim 300 \mathrm{pc}$. Both the molecular clouds in the $1.7 \mathrm{pc}$ ring and the ionized streamers within 1 pc have $n_{\text {pre }} \sim 10^{4}-10^{5} \mathrm{~cm}^{-3}$, much less than $n_{\text {Roche }}$. However, if the clouds or streamers collided with their characteristic velocities $v_{\text {rel }} \simeq 100 v_{s 7} \mathrm{~km} \mathrm{~s}^{-1}$, the shocked gas would quickly cool to $\sim 10^{4} \mathrm{~K}$, and more slowly, but still within a collision time, to $T_{f} \sim 40 \tau^{-1 / 4} V_{s 7}^{3 / 4} \mathrm{~K}$, where $\tau$ is the dust optical depth at the peak wavelength of dust emission. The post-shock density is then

$$
n_{\text {post }} \sim 10^{9}\left(n_{\text {pre }} / 10^{4} \mathrm{~cm}^{-3}\right) v_{s 7}^{5 / 4} \tau^{1 / 4} \mathrm{~cm}^{-3},
$$

which amply exceeds the Roche density, and is gravitationally unstable. The column density of clumps in the $1.7 \mathrm{pc}$ molecular ring is $\sim 10^{23.5} \mathrm{~cm}^{-2}$, while that of the ionized streamers $\sim 10^{22.3} \mathrm{~cm}^{-2}$. At the Roche density, $1 M_{\odot}$ occupies a region $\sim 10^{16.3} r_{p c}^{2 / 3} \mathrm{~cm}$ in size, and has column density $N \sim 10^{24} r_{p c}^{-4 / 3} \mathrm{~cm}^{-2}$. Thus collisions between the molecular clumps could easily result in formation of stars; star formation from the ionized gas is marginal. If collapse begins, it can form a condensed protostar even as the collision proceeds, since the accretion timescale $\sim M / \dot{M} \sim M c_{s}^{3} / G \sim 10^{4} T_{2}^{-3 / 2} \mathrm{yr}$.

We conclude that the molecular torus may be forming stars, but that the interior ionized gas may be destined to collect in the center (unless ejected).

\subsection{PROBLEMS OF KEEPING THE TORUS PUMPED UP}

The $1.7 \mathrm{pc}$ molecular ring has substantial thickness $(h / r \gtrsim 0.3)$ and velocity dispersion $(\sigma \gtrsim$ $30 \mathrm{~km} \mathrm{~s}^{-1}$, Genzel \& Townes 1987). Without an energy source, collisions and the resulting radiative cooling would collapse the torus within a few orbital periods $\left(P_{\text {orb }} \sim 10^{4} \mathrm{yr}\right.$ ). A similar problem occurs in molecular clouds (supersonic turbulence) and in the $\sim 1$ pc molecular tori of Seyfert galaxies (Krolik \& Begelman 1988). The hackneyed explanations are that the gas is pumped up by winds or tidal ejecta from stars, or by tapping the energy of bulk differential rotation of the torus. The former is inefficient because winds couple only with efliciency $\sigma / v_{w}$, so keeping the torus pumped up would require a star formation or disruption rate $\gtrsim 10^{-4}\left(v_{w} / \sigma\right) M_{\odot} \mathrm{yr}^{-1}$, barely consistent with the Copernican principle. Tapping shear energy works only if the molecular clumps have coefficients of restitution like tennis balls ( $\gtrsim 0.3$, Krolik \& Begelman 1988), counter intuitive, but perhaps possible if strong magnetic fields are embedded. In our Galactic Center, we have a unique opportunity to diagnose both magnetic fields and stellar activity, and thus shed light on the nature of the nuclear tori in Seyfert galaxies. 


\section{Conclusions}

Unless most of the cooling gas in the Galactic Center is somehow ejected, it is hard to see how $a \gtrsim 10^{5} M_{\odot}$ black hole can have avoided forming. Within $0.2 \mathrm{pc}$, stars are merged, not born. The black hole, if present, is well disguised, but could shine forth at any time.

I thank: the editor for his forbearance, Liz for typing, Tim de Zeeuw for comments, and the Irvine Foundation and the NSF for support under PYI grant AST 84-51725.

\section{References}

AIP 155: The Galactic Genter, ed. D.C. Backer (Am. Inst. Phys. Conf. Proc. No. 155, AIP, N.Y.)

AlleI, D.A. 1987, AIP 155, 1.

Backer, D.C., and Sramek, R.A. 1987, AIP 155, 163.

Bahcall, J.N., and Wolf, R.A. 1976, Ap. J., 209, 214.

Bailey, M.E., 1980, M. N. R. A. S., 190, 217.

Becklin, E.E., and Neugebauer, G. 1968, Ap. J., 151, 145.

Begelman, M.C., and Rees, M.J. 1978, M. N. R. A. S., 185, 847

Begelnuan, M.C., Sikora, M., and Rees, M.J. 1987, Ap. J., 313, 689.

Coln, H. 1980, Ap. J., 242, 765.

Colgate, S.A., 1967, Ap. J., 150, 163.

Dressler, A., and Riclistone, D. 1988, Ap. J., 324, 701.

Engelke, C!W., 1988, Ap. J., $325,153$.

Forrest, W.J., Shure, M.A., Pipher, J.L., and Woodward, C.E. 1987, AIP 155, 153.

Gatley, I. 1987, AIP 155, 8.

Geballe, T.R., Wade, R., Krisciunas, K., Gatley, I., and Bird, M.C. 1987, Ap. J., 320, 562.

Genzel, R., and Townes, C.H. 1987, Ann. Rev. Astr. Ap., 25, 377.

Giisten, R. 1987, AIP 155,19.

Hills, J.G., 1988, Nature, $331,687$.

Inngaki, S., and I.ynden-Bell, D. 1983, M. N. R. A. S., $205,913$.

Inagaki, S., and Saslaw, W.C. 1985, Ap. J., $292,339$.

Jauncey, D.L. et al. 1988, JPL astrophysics preprint 155

Kaw ai, N. et $u$ l. 1988, $A$ p. J., 330, 130.

Keel, W.C. 1985, in Astrophysics of Active Galaxies, ed. J.S. Miller (University Science Books, Mill Valley), 1.

Kormendy, J. $1988 a$, Ap. J., 325, 128.

Kormendy, J. 1988b, Ap. J., 335, 40.

Kluźniak, W., Ruderman, M., Shaham, J., and Tavani, M. 1988, Nature, 336, 558.

Krolik, J.H., and Begelman, M.C., 1988, Ap. J., 329, 702.

Lacy, J.H., Townes, C.H., and Hollenbach, D.J. 1982, Ap. J., 262, 120.

Lebofsky, M.J., and Rieke, G.H. 1987, AIP 155, 79.

Lee, H.M., and Nelson, L.A. 1988, Ap. J., 334, 688.

Lightman, A.P., Zdziarski, A.A., and Rees, M.J. 1987, Ap. J., 315, I113.

Lingenfelter, R.E., and Ramaty, R. 1983, in Positron-Electron Pairs in Astrophysics, eds. M.L. Burns, A.K. Harding, R. Ramaty (AIP Conf. Proc. No. 101, AIP, N.Y.), 267.

McGinn, M.T., Sellgren, K., Becklin, E.E., and Hall, D.N.B. 1988 preprint.

Murphy, B.W., and Cohn, H.N. 1988, M. N. R. A. S., 232, 835.

Plinney, E.S. 1983, Ph. D. thesis, Cambridge University.

Ramaty, R., and Lingenfelter, R.E. 1987, AIP 155, 51.

Rees, M.J., Begelman, M.C., Blandford, R.D., and Phinney, E.S. 1982, Nature, $295,17$.

Rees, M.J. 1987, AIP 155, 71.

Rees, M.J. 1988, Nature, 333, 523.

Renzini, A., and Buzzoni, A. 1986, in Spectral Evolution of Galaxies, eds. C.Chiosi \& Renzini, A., (Dordrecht, Reidel), 195 .

Reynolds, S.P., and McKee, C.F. 1980, Ap. J., $239,893$.

Rieke, G.H., and Rieke, M.J. 1988, Ap. J., 330, L33.

Sanders, R.H. 1970, Ap. J., 162, 791.

Serabyn, E., and Lacy, J.H., 1985, Ap. J., 293, 445.

Share, G.H. et al. 1988, Ap. J., 326, 717.

Skinmer, G.K. et al. 1987, Nature, 330, 544.

'Tonry, J. 1987, Ap. J., 322, 632.

Vauclen Berg, D.A., and Laskarides, P.G. 1987, Ap. J. Suppl., 64, 103.

Wacle, R., Geballe, T.R., Krisciunas, K., Gatley, I., and Bird, M.C. 1987, Ap. J., 320, 570. 